\title{
Morfología Microestructural Dérmica en Juveniles de Dos Es- pecies Hermanas de Tiburón Pala, Sphyrna tiburo y S. vespertina
}

\author{
Dermal Microstructural Morphology in Juveniles of Two Sister \\ Species of Bonnethead Shark, Sphyrna tiburo and S. vespertina
}

Luis Brain Carrasco-Martínez ${ }^{1}$ Luis Fernando Del Moral-Flores ${ }^{1}$; Rafael Emiliano Quintanar-Zúñiga ${ }^{2}$ \& Francisco Sancho-Vázquez ${ }^{3}$

CARRASCO-MARTÍNEZ, L. B.; DEL MORAL-FLORES, L. F.; QUINTANAR-ZÚÑIGA, R. E. \& SANCHO-VÁZQUEZ, F. Morfología microestructural dérmica en juveniles de dos especies hermanas de tiburón pala, Sphyrna tiburo y S. vespertina. Int. J. Morphol., 39(4):1160-1163, 2021.

RESUMEN: Los dentículos dérmicos son estructuras dermales presentes en el grupo de los condrictios, tienen un papel muy importante en su biología y se les ha utilizado como un carácter taxonómico que permiten reconocer grupos o especies. Por lo que en el presente trabajo se compara la morfología dermal de los juveniles de dos especies de tiburones pala, Sphyrna tiburo y $S$. vespertina, cuyo origen evolutivo está emparentado con el cierre del istmo centroaméricano. Para ello se obtuvieron muestras dermales $\left(1 \mathrm{~cm}^{2}\right)$ de tres regiones corporales y se procesaron para obtener imágenes de alta resolución por medio de Microscopia electrónica de barrido (MEB). Los dentículos de ambas especies tienen un patrón morfológico común, con variaciones en la longitud de las prolongaciones de las crestas, área libre y superposición de los dentículos, y grado de notoriedad de la ornamentación microestructural.

PALABRAS CLAVE: Dentículo dérmico; Elasmobranquios; Microscopía electrónica de barrido; Sphyrnidae; Escama.

\section{INTRODUCCIÓN}

Los tiburones de la familia Sphyrnidae (Carcharhiniformes: Elasmobrancii), son conocidos comúnmente como cornudas, tiburones martillo o pala. Su nombre deriva por la forma de sus cabezas, debido a la expansión lateral de las cápsulas nasales, presencia de ojos en los extremos laterales y el aplanamiento dorso-ventralde su cabeza (Compagno, 1988). Esta es una sinapomorfia del grupo, considerado monofilético, el cual guarda parentesco con la familia Carcharhinidae (Naylor, 1992). A pesar de los amplios estudios filogenéticos al interior de la familia, aún no se esclarecen por completo las relaciones entre géneros y especies. Para ello, se han considerado datos morfológicos (Gilbert, 1967; Compagno), moleculares (Naylor; Martin, 1993; Lim et al., 2010) y una combinación de ambos (Cavalcanti, 2007).

El clado de los pequeños tiburones pala, se caracteriza por tener un menor desarrollo lateral de la cabeza, aparentan ser un grupo derivado (Lim et al.). Entre ellas, las especies Sphyrna tiburo (Atlántico occidental) y $S$. vespertina (Pacífico oriental) fueron consideradas como una sola especie debido a su gran similitud morfológica (Bigelow \& Schroeder, 1948). Sin embargo, existen diferencias a nivel cefálico (Gilbert). En la actualidad son consideras especies válidas, debido a su distribución disyunta (Del Moral-Flores et al., 2015). El origen anfiamericano de estas especies hermanas, se estima al Plioceno tardío, durante la emergencia del Istmo centroaméricano (Lim et al.).

Debido a la similitud morfológica de varias especies de tiburones, se han buscado características y estructuras que ayuden a su reconocimiento. Destacan los dientes y los dentículos dérmicos, estos últimos también son llamados escamas placoideas, juntos han sido una herramienta útil en los estudios paleontológicos por su fuerte mineralización y preservación en el registro fósil (Feichtinger et al., 2020). En el caso de los dentículos

${ }^{1}$ Laboratorio de Zoología, Facultad de Estudios Superiores Iztacala, Universidad Nacional Autónoma de México (UNAM), Av. de los Barrios No. 1, Los Reyes Iztacala, C.P. 54090 Tlalnepantla, Estado de México, México.

${ }^{2}$ Unidad de Biotecnología y Prototipos (UBIPRO), Facultad de Estudios Superiores Iztacala, UNAM, Av. de los Barrios No. 1, Los Reyes Iztacala, C.P. 54090 Tlalnepantla, Estado de México, México.

${ }^{3}$ Centro de Estudios Tecnológicos del Mar No. 24, Puerto Madero, Chiapas, México. 
dérmicos, tienen la ventaja de mantener su integridad morfológica y permiten una relación con las especies. Las principales funciones de los dentículos dérmicos son la protección, defensa, acoplamiento de órganos sensoriales, flujo hidrodinámico, entro otros (Raschi \& Tabit, 1992).

El estudio de los dentículos dérmicos ha cobrado gran importancia, y la microscopia electrónica de barrido (MED) ha permitido describir y analizar a mayor profundidad su morfología microestructural (Dingerkus \& Koestler, 1986). Estos estudios han apoyado los programas de conservación, al determinar las especies sujetas al aleteo o "finning" (Tanaka et al., 2002). Sin embargo, se han observado variaciones de los dentículos dependiendo de la región corporal del tiburón, y puede ocasionar problemas en la identificación de las especies. A pesar de la gran cantidad de información sobre dentículos dérmicos, pocos han evaluado las relaciones que guardan entre congéneres. De manera particular, Mello et al. (2011), analizan la diversidad microestructural de seis especies de la familia Sphyrnidae en estado prenatal, neotanal y juvenil, incluyendo a $S$. tiburo. Sin embargo, no encontraron diferencias entre sexos, ni claras diferencias entre regiones corporales, además, a pesar de encontrar un patrón morfológico entre las especies de mayor tamaño y las de tamaño pequeño, no encontraron características que permitan reconocer a cada una de las especies de esfírnidos estudiadas. Sin embargo, aún faltan varias especies de dicha familia por analizar, cuya implicación evolutiva pueda ayudar a comprender la similitud de los dentículos dérmicos. Es por lo que en la presente contribución se describen y compara la morfologíade los dentículos entre juveniles de las especies hermanas de tiburones pala, Sphyrna tiburo y $S$. vespertina.

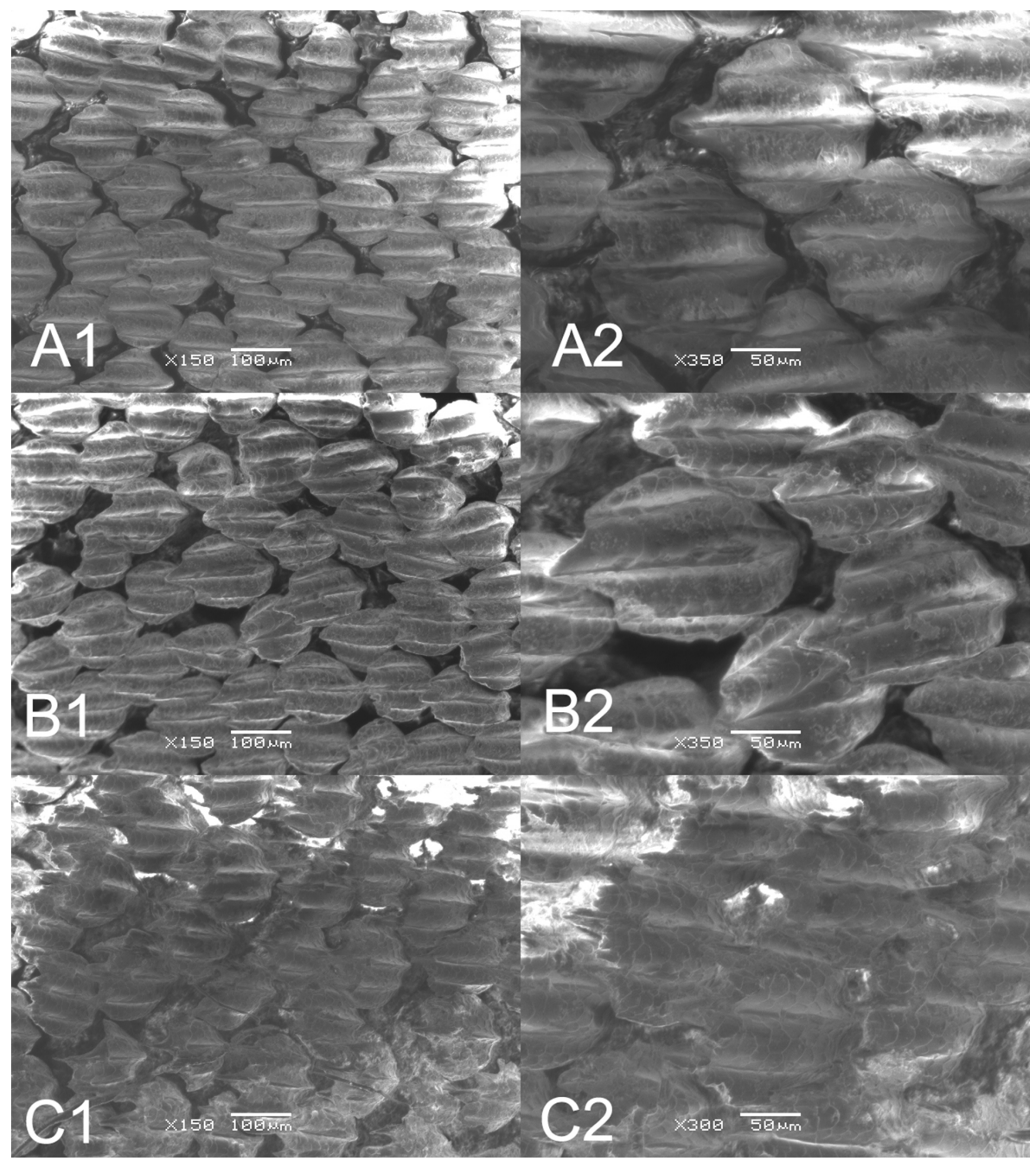

Fig. 1. Dentículos dérmicos en microscopio electrónico de barrido de Sphyrna tiburo: A) Ventrales de la región cefálica (A1 X150, A2 X400); B) Dorsales de la región cefálica (B1 X150, B2 x400); C) Región dorsal (C1 X150, C2 x350).

\section{MATERIAL Y MÉTODO}

Las muestras de piel se obtuvieron de 29 especímenes neonatos y juveniles de ambas especies de tiburones martillo que se encuentran depositados en la Colección Ictiológica de Facultad de Estudios Superiores Iztacala (CIFI): Sphyrna tiburo $(\mathrm{n}=15 ; 213-430 \mathrm{~mm}$ de longitud total (LT)), y $S$. vespertina $(\mathrm{n}=14 ;$ 585-670 mm LT). Para ello, diseccionaron aproximadamente $2 \mathrm{~cm}^{2}$ de piel de tres regiones corporales: región cefálica dorsal (entre las órbitas oculares); región cefálica ventral (entre la región internasal) y en la base de la aleta dorsal. Cada muestra dérmica fue deshidratada mediante una serie gradual de etanol $\left(40^{\circ}, 70^{\circ}, 96^{\circ}\right)$ por períodos de $24 \mathrm{~h}$ (Sandoval et al., 2016), se dejaron secar a la intemperie y posteriormente se obtuvo $1 \mathrm{~cm}^{2}$ y se montó en una placa de aluminio que fue recubierta con oro, mediante pulverización iónica y se tomaron imágenes con un microscopio electrónico de barrido (MEB) (Fig. 1). 


\section{RESULTADOS}

De manera general, los dentículos dérmicos en ambas especies presentan tamaños pequeños, entre 100-120 $\mu \mathrm{m}$ entre la parte anterior y posterior de corona; la superficie dorsal de la corona tiene un patrón romboidal, el margen anterior es crenado y más ancho que el posterior. El margen posterior es aserrado, por la prolongación de las tres quillas o crestas centrales que corren paralelamente entre sí; la longitud y altura de las crestas varía entre las regiones corporales, pero la central tiende a ser la más larga, formando una cúspide central. El ancho de las alas laterales es menor o igual a la que existe entre las crestas laterales y central. Se presenta sobre la superficie dorsal cubierta por una ornamentación microestructural que se distingue solo con MEB, son de forma convexa hacia el margen anterior y sus bordes no son muy marcados.

Se observaron los dos tipos de arreglos dérmicos reportados por Mello et al., entre mezclados, una mayor área es dominada por inserción lateral y en menor medida la superposición. Aunque se observó una ligera variación entre las regiones corporales de ambas especies, la región dorsal presentó una menor superposición de los dentículos, así como una menor longitud en la proyección posterior de las crestas.

No se observaron diferencias entre sexos, pero al hacer las comparaciones entre $S$. tiburo y $S$. vespertina se encontraron algunas diferencias entre los dentículos dérmicos. Los dentículos de $S$. tiburo presentaron una mayor prolongación en el borde posterior de las crestas, su corona es más compacta; además, presentó una menor área libre entre los dentículos de las tres regiones. Por otra parte, la corona de $S$. vespertina es más amplia, y la altura de sus crestas es menor; en esta especie la ornamentación microestructural no fue tan marcada en comparación con $S$. tiburo. (Figs. 1 y 2)

\section{DISCUSIÓN}

A pesar de existir un patrón en la morfología de los dentículos dérmicos en las especies de tiburón martillo bajo estudio, existe una ligera variación entre regiones corpora-

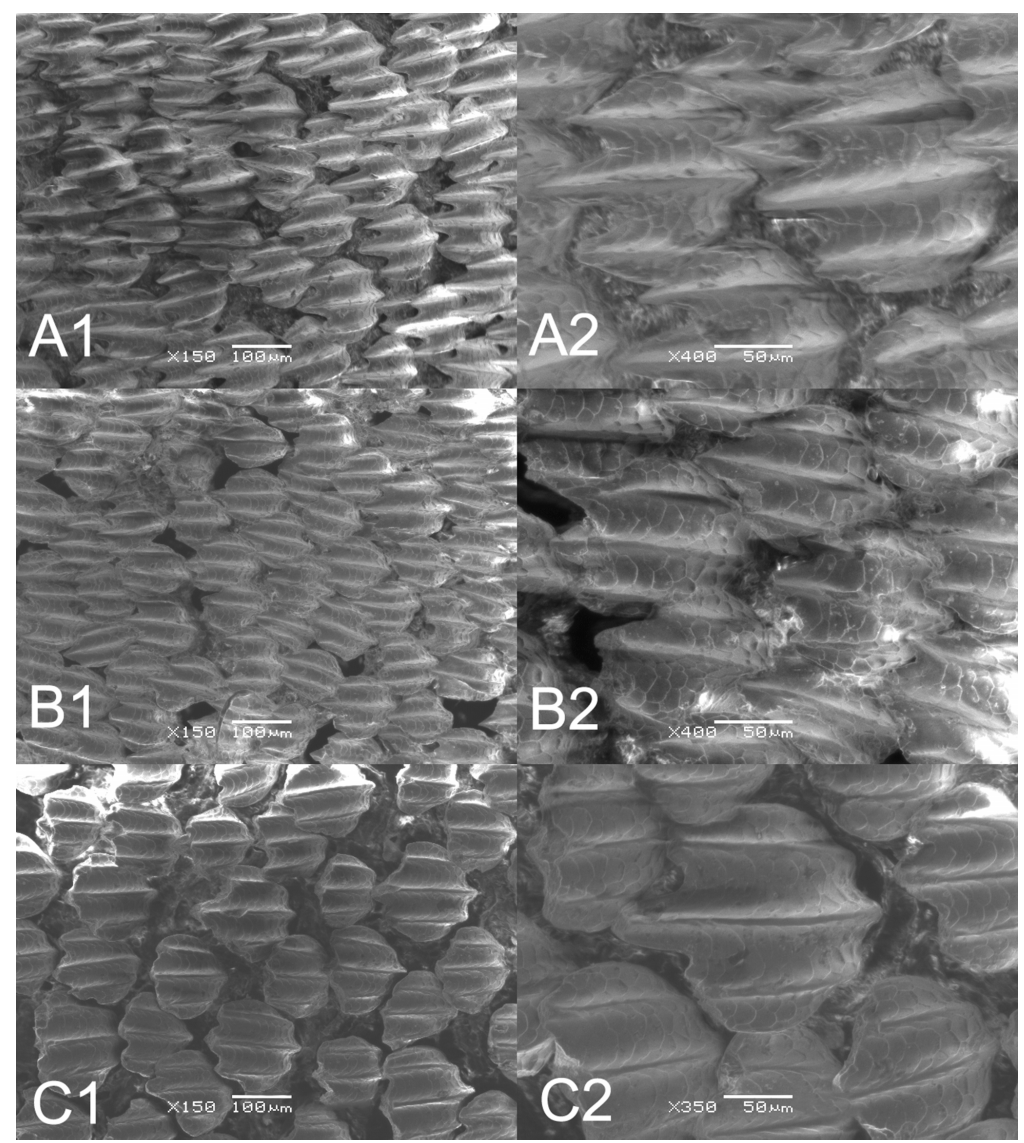

Fig. 2. Dentículos dérmicos en microscopio electrónico de barrido de Sphyrna vespertina: A) Ventrales de la región cefálica (A1 X150, A2 X350); B) Dorsales de la región cefálica (B1 X150, B2 x350);C) Región dorsal (C1 X150, C2 X300).

les, esto sugiere una relación morfológica de la escama y su funcionalidad. Se ha comprobado que en algunas especies de tiburones de hábitos costeros y de profundidad hay una ampla variación en tamaño, forma y espaciado de los dentículos, conforme a determinadas regiones corporales, incluyendo los dentículos orales (Ankhelyi et al., 2018; Rangel et al., 2018). Por lo que es necesario analizar con mayor detalle otras regiones corporales.

Los dentículos tienen diversas funciones específicas, suelen agruparse en distintos morfotipos funcionales, como los de protección, reducción de fricción y algunas funciones generalizadas enmarcadas por la ecología de cada especie (Raschi \& Tabit). En ambas especies, el morfo tipo tricúspide fue el más frecuente y abundante, está relacionado con la reducción de la resistencia hidrodinámica, pues se sabe que la presencia de las crestas disminuye las turbulencias a medida que el agua fluye y el tiburón se desplaza (Dillon et al., 2017). Sin embargo, es posible que el tamaño y densidad de los dentículos les brinden protección, debido a que son especies de hábitos costeros y tamaño pequeño (Compagno). 
La identificación taxonómica entre estas dos especies puede estar reforzada con un estudio detallado de los dentículos dérmicos, pues con base en nuestras observaciones podemos apreciar patrones distintivos entre una especie y otra en la región cefálica. Se sabe que estás dos especies de tiburón martillo presentan unos dentículos dérmicos más reducidos en comparación con las especies de mayor tamaño que presentan una distribución circunglobal (Mello et al.). Este patrón morfológico, al igual que el cambio ontogénico de las cúspides de los dientes anteriores (Mello $\&$ Machado Brito, 2013), entre otras, puedan ser una señal de su parentesco filogenético.

\section{AGRADECIMIENTOS}

Al SIN-CONACYT y al proyecto PAPIIT-UNAM IA207820 por el financiamiento proporcionado. Extendemos nuestro agradecimiento a la cooperativa pesquera de Puerto Madero, Chiapas, por su apoyo en la obtención de ejemplares.

\section{CARRASCO-MARTÍNEZ, L. B.; DEL MORAL-FLORES,} L. F.; QUINTANAR-ZÚÑIGA, R. E. \& SANCHOVÁZQUEZ, F. Dermal microstructural morphology in juveniles of two sister species of Bonnethead shark, Sphyrna tiburo and S. vespertina. Int. J. Morphol., 39(4):1160-1163, 2021.

SUMMARY: The dermal denticles are dermal structures present in the group of chondrichthyans, they have a very important role in their biology and they have been used as a taxonomic character that allows to recognize groups or species. Therefore, in the present work, the dermal morphology of the juveniles of two species of shovel sharks, Sphyrna tiburo and $S$. vespertina, whose evolutionary origin is related to the closure of the Central American isthmus, is compared. For this, dermal samples $\left(1 \mathrm{~cm}^{2}\right)$ from three body regions were obtained and processed to obtain high resolution images by means of scanning electron microscopy (SEM). The denticles of both species have a common morphological pattern, with variations in the length of the ridge extensions, free area and overlapping of the denticles, and the degree of notoriety of the microstructural ornamentation.

KEY WORDS: Dermal denticle; Elasmobranch; Spyrnidae; Scale; Scanning electron microscopy.

\section{REFERENCIAS BIBLIOGRÁFICAS}

Ankhelyi, M.; Wainwright, D. K. \& Lauder, G. V. Diversity of dermal denticle structure in sharks: skin surface roughness and three-dimensional morphology. J. Morphol., 279(8):1132-54, 2018.
Bigelow, H. \& Schroeder, W. Sharks. In: Tee-Van, J.; Breder, C. M.; Hildebrand, S. F.; Parr, A. E. \& Schroeder, W. C. (Eds.). Fishes of the Western North Atlantic. Part I, Vol. I, Memoirs of the Sears Foundation for Marine Research, 1(1):59-576, 1948.

Cavalcanti, M. A phylogenetic supertree of the hammerhead sharks (Carcharhiniformes: Sphyrnidae). Zool. Stud., 46(1):6-11, 2007.

Compagno, L. J. V. Sharks of the Order Carcharhiniformes. New Jersey, Princeton University Press, 1988.

Del Moral-Flores, L. F.; Morrone, J. J.; Alcocer-Durand, J.; Espinosa Pérez, H. \& Pérez-Ponce De León, G. Lista patrón de los tiburones, rayas y quimeras (Chondrichthyes, Elasmobranchii, Holocephali) de México. Arx. Misc. Zool., 13:47-163, 2015.

Dillon, E. M.; Norris, R. D. \& Dea, A. O. Dermal denticles as a tool to reconstruct shark communities. Mar. Ecol. Prog. Ser., 566:117-34, 2017.

Dingerkus, G. \& Koestler, R. J. Application of scanning electron microscopy to the study of shark dermal denticles. Scan. Elect. Mic., 2:513-9, 1986.

Feichtinger, I.; Lukeneder, A. \& Guinot. G. A lower Cretaceous chondrichthyan dermal denticle assemblage and its bearing on placoid scale diversity and histology. Cretac. Res., 111:1-17, 2020.

Gilbert, C. A revision of the hammerhead sharks (Family Sphirnidae). Proc. U. S. Natl. Mus., 119(3539):1-88, 1967.

Lim, D. D.; Motta, P.; Mara, K. \& Martin, A. P. Phylogeny of hammerhead sharks (Family Sphyrnidae) inferred from mitochondrial and nuclear genes. Mol. Phylogenet. Evol., 55(2):572-9, 2010.

Martin, A. Hammerhead shark origins. Nature, 364:494, 1993.

Mello, W. \& Machado Brito, P. M. Contributions to the tooth morphology in early embryos of three species of hammerhead sharks (Elasmobranchii: Sphyrnidae) and their evolutionary implications. $C$. R. Biol., 336(9):466-71, 2013.

Mello, W. C.; de Carvalho, J. J. \& Brito, P. M.M. Microestructural morphology in early dermal denticles of hammerhead sharks (Elasmoranchii: Sphyrnidae) and related taxa. Acta Zool., 94(2):14753, 2011.

Naylor, G. J. P. The phylogenetic relationships among réquiem and hammerhead sharks: inferring phylogeny when thousands of equally most parsimonious tree results. Cladistics, 8(4):295-318, 1992.

Raschi, W. \& Tabit, C. Functional aspects of Placoid Scales: A review and update. Aust. J. Mar. Freshw. Res., 43(1):123-47, 1992.

Sandoval, D.; Téllez, J.; García, A.; Rivera, G.; Moreno, S. \& Moreno, F. Técnica de diafanización para describir el desarrollo embrionario del sistema óseo: revisión de la literatura. Univ. Med., 57(4):488-501, 2016.

Tanaka, S.; Kitamura, T. \& Nakano, H. Identification of shark species by SEM observation of denticle of shark fins. ICCAT, 54(4):1386-94, 2002.

\section{Dirección para correspondencia}

Laboratorio de Zoología

Facultad de Estudios Superiores Iztacala

Universidad Nacional Autónoma de México

Av. de los Barrios No. 1

Los Reyes Iztacala

C.P. 54090 Tlalnepantla

Estado de México

MÉXICO

E-mail: delmoralfer@comunidad.unam.mx

Recibido : 27-04-2021

Aceptado: 26-05-2021 